\title{
The incidence of postoperative pulmonary perfusion defects
}

\author{
N. J. GRIFFITHS* \\ F.R.C.S. \\ R. J. WightoN $\dagger$ \\ M.Sc. \\ J. B. Festenstein \\ F.F.R. \\ ${ }^{*}$ Departments of Surgery, and †Medical Physics, North Middlesex Hospital, Edmonton
}

\begin{abstract}
Summary
The incidence of postoperative thromboembolism was studied using radioisotope lung perfusion and ventilation scanning. $26 \%$ of patients had defects on their pre-operative scans and $8 \%$ developed new unmatched perfusion defects postoperatively, indicating possible pulmonary emboli. This latter figure was much lower than some previously recorded results and was similar in 2 groups of patients - one receiving dextran 70 prophylaxis during operation and the other a control group who received saline.
\end{abstract}

\section{Introduction}

Post-mortem studies have revealed the true incidence of unsuspected deep venous thrombosis (DVT) and pulmonary embolism following operation, Wessler (1961) found pulmonary embolism in $70 \%$ of his cases, and Morrell and Dunhill (1968) who looked at 263 right lungs found evidence of pulmonary embolism in $51.7 \%$ and this being the only cause of death in $14 \%$.

With the advent of the fibrinogen uptake test the frequency of deep venous thrombosis following operation has been shown to be $25-30 \%$. Nicolaides and Irving (1975) in a study of 626 patients found the incidence of isotopic deep vein thrombosis to be $26 \%$ of patients undergoing general surgical operations, and as high as $49 \%$ in patients who underwent hip surgery. Kakkar et al. (1970) suggested that certain patients preparing for surgery were at 'great risk' of developing DVT, namely those with a previous history of pulmonary emboli and deep venous thrombosis, operations on varicose veins, obesity, malignancy and old age. With this awareness have come various methods of prophylaxis in an attempt to prevent DVT and subsequent pulmonary emboli, and 2 clinical trials, one using dextran 70 (Kline et al., 1975) the other, subcutaneous heparin (International Multicentre Trial, 1977), both demonstrated a significant reduction in

* Present address : Department of Surgery, St Bartholomew's Hospital, London, E.C.1. the incidence of fatal pulmonary emboli in the treated groups.

The combination of radioisotope lung perfusion of and ventilation scintigraphy has been shown to be accurate in the diagnosis of pulmonary embolism in (Williams et al., 1974).

It has been suggested that with lung perfusion 을 and ventilation scintigraphy the incidence of post- operative thromboembolism is $18-24 \%$ (Browse, $\rightarrow$ Clemenson and Croft, 1974; Browse et al., 1976). 을. Furthermore, Browse showed that the use of dextran $\overrightarrow{0}$ 70 and pneumatic leg compression during surgetg reduced the incidence of postoperative lung perfusion defects from $24 \%$ in the control group to $8 \%$ in the group given the dextran 70 and pneumatic leg compression prophylaxis (Browse et al., 1976).

The present authors looked at the incidence of postoperative unmatched lung perfusion defects $\triangle$ following surgery and the possible effect of dextran 70 used alone as prophylaxis in the prevention of thromboembolism.

\section{Patients and methods}

Fifty-eight patients undergoing major abdominal surgery were allocated by the use of random numbers to either a group receiving dextran 70 or a group receiving saline during surgery. The amount of dextran given was that described by Kline et al. (1975), $500 \mathrm{ml}$ of dextran 70 were given after induction of anaesthesia and a further $500 \mathrm{ml}$ over a $>$ period of more than $2 \mathrm{hr}$ after the completion of the operation. The other group of patients received a $N$ similar amount of normal saline.

After informed consent had been obtained, $\tilde{O}$ ventilation and perfusion scans were performed on $\mathrm{c}$ the day before surgery and then repeated on the $\underset{T}{ }$ sixth postoperative day.

Lung ventilation studies were performed using the radioactive gas ${ }^{81 \mathrm{~m}} \mathrm{Kr}$ (Fazio and Jones, 1975).

Lung perfusion was assessed using $1 \mathrm{mCi}$ ${ }^{99 m}$ Tc-labelled macro-aggregated albumin injected intravenously with the patient supine. 
With the patient seated, anterior, posterior and lateral views were imaged using a Radicamera Mark II gamma camera and the data displayed via a digital equipment corporation gamma 11 system.

The scans were viewed independently by a radiologist and a physicist, neither of whom knew the clinical or surgical history of the patient. A thromboembolism was diagnosed if a new perfusion defect was found postoperatively in the presence of an unchanged ventilation scan (unmatched perfusion defect).

The first 13 patients had pre- and postoperative perfusion scans only. These were performed on a DS7-W Selo rectilinear scanner with the patient supine.

All the patients had pre-operative chest X-ray, none of which demonstrated any evidence of previous lung pathology.

\section{Results}

Fifty-eight patients had pre-operative lung scans, but 7 patients were unable or refused to have postoperative lung scans and were excluded from the study. Also excluded from the study. were any patients with a previous history of deep venous thrombosis or thromboembolism. From the remaining 51 patients 25 were given dextran 70 prophylaxis and 26 a similar amount of saline. The distribution of age, sex, whether the patient had benign or malignant disease and length of operation was similar in both groups (Table 1).

The first 6 patients in the dextran group and the first 6 controls has perfusion scans only.

Thirteen patients $(26 \%)$ had an abnormality in the ventilation or perfusion scan pre-operatively, 6 patients perfusion only ( 2 had perfusion scans only), 2 ventilation defects only and 5 a combination of ventilation and perfusion defects.

Following operation, 3 of the ventilation defects improved, in 2 the accompanying perfusion defect did not alter whilst in the third the perfusion defect also disappeared. Likewise 2 patients who had perfusion scans only and had demonstrated defects before operation had normal scans following operation.

There was no clinical evidence of deep venous thrombosis or pulmonary embolism in any of the patients studied.

Nine patients received blood transfusion during or after surgery, 5 in the dextran group and 4 in the control group, the average amount of blood transfused was 1.5 litre. Only one of the 4 patients who had a new postoperative perfusion defect received a blood transfusion.

Of the 7 patients who did not have postoperative lung scans, 3 refused and in all these there were no clinical signs to indicate deep venous thrombosis or pulmonary embolism; the remaining 4 died at varying times in the postoperative period and all had post-mortem examinations where no evidence of pulmonary emboli was found.

\section{Discussion}

When they were scanned pre-operatively, $26 \%$ of patients had an abnormality of either perfusion or ventilation. This figure is in agreement with other workers (Browse et al., 1976) and again demonstrates the importance of pre-operative scanning when assessing postoperative perfusion or ventilation lung scanning. The pre-operative defects were evenly distributed between perfusion, ventilation and combination defects and in all the patients no abnormalities were detected on chest X-ray. Preoperatively 6 patients had perfusion defects and in 4 of these in whom ventilation scanning was performed no defects were found. There was no previous history of deep venous thrombosis or pulmonary embolism in any of these patients. This suggests that the results of isolated perfusion scanning should be treated with caution and that a normal scan may be more helpful than an abnormal scan in certain cases where the diagnosis of pulmonary embolism is in doubt, in that it excludes thromboembolism.

Postoperatively 9 patients still had an abnormality on ventilation or perfusion scanning, those patients who had abnormal ventilation scans but normal perfusion scans pre-operatively had normal scans one week after operation. This is believed to be a result of postoperative physiotherapy and, unlike Aukland, Coates and Hirsch (1977), the authors found no new ventilation defects in this series. Likewise, 3 perfusion defects returned to normal

TABLE 1. Illustrating patients admitted to a trial comparing the group receiving dextran 70 prophylaxis with a control group

\begin{tabular}{lcccccc}
\hline $\begin{array}{l}\text { Treatment } \\
\text { (no. of } \\
\text { patients) }\end{array}$ & $\begin{array}{c}\text { Average } \\
\text { age } \\
\text { (years) }\end{array}$ & Male & Female & Benign & Malignant & $\begin{array}{c}\text { Average } \\
\text { length of } \\
\text { operation } \\
\text { (min) }\end{array}$ \\
\hline Dextran (25) & 52 & 11 & 14 & 23 & 2 & 72 \\
Saline (26) & 52 & 10 & 16 & 20 & 6 & 67 \\
\hline
\end{tabular}


postoperatively, only one being associated with a ventilation defect which also returned to normal.

There was a similar incidence of pre- and postoperative lung scanning abnormalities in the dextran 70 and the control group.

Following operation, there were 4 new unmatched perfusion defects, 2 in the group receiving dextran 70 and 2 in the control group, an incidence in each case of $8 \%$. This is a much lower figure than previous workers have reported (Browse et al., 1974, 1976) but is in agreement with Knight and Metrevelli (1977). The low incidence in Knight and Metrevelli's patients might be explained by the fact that 11 of the 26 patients studied underwent hernia repair, a group of patients in whom thromboembolism rarely occurs. In the present series, however, every patient had muscle relaxant anaesthesia and a laparotomy. A common denominator between the 2 series with a low incidence of postoperative thromboembolism was the use of ${ }^{81 \mathrm{~m}} \mathrm{Kr}$ instead of ${ }^{133} \mathrm{Xe}$ for the ventilation studies but the authors feel that in view of the low incidence of both perfusion and ventilation defects following operation the use of krypton did not influence the results. Whether $8 \%$ reflects the true incidence of postoperative thromboembolism is uncertain, because minor thromboembolisms are most likely cleared rapidly from the lung by the fibrinolytic activity generated by the pulmonary arteries, in fact, Knight and Metrevelli found a higher incidence on the third postoperative day when compared with the sixth postoperative day.

Dextran 70 did not appear to influence the development of unmatched perfusion defects as the incidence was the same in both the dextran-treated and the control group but the numbers in each group were too small to draw firm conclusions.

In previous studies long pre-operative stay in hospital and the length of operation have been cited as possible relevant factors in the aetiology of postoperative emboli. The majority of patients in the present series were admitted $36 \mathrm{hr}$ before operation and in very few was the length of operation prolonged (Table 1). Consequently these 2 factors may be relevant in explaining the low incidence of unmatched perfusion defects in this series. Other factors which could also be important are the relative low incidence of patients with malignant disease ( 8 of 51 ), the absence of patients with a previous history of DVT or pulmonary embolism, and the average age of 52 years, although the span was 32-76 years. There is no evidence that any of these patients were excessively obese or had significant varicose veins, further factors which Kakkar et al. (1970) suggested might be of importance in selecting a high risk group who might develop postoperative DVT and possibly pulmonary emboli. The authors feel, however, that these patients, all of whom underwent a laparotomy, are a true reflection of patients entering a surgical unit of a district general hospital.

Only one of the 4 new postoperative unmatched perfusion defects received a blood transfusion compared with 5 patients who received dextran 70 prophylaxis (average amount 1.0 litre blood), consequently it is unlikely that a blood transfusion had any connection with the development of a thromboembolism as detected by combined perfusion ventilation scanning.

Consequently the authors are unable to explain the low incidence of unmatched postoperative perfusion defects in this series but would agree with Knight and Metrevelli that the incidence of postoperative thromboembolism as determined by lung scanning is much lower than previously reported, and that it remains to be seen whether dextran 70 prophylaxis can prevent these thomboemboli.

\section{Acknowledgments}

We would like to thank $\mathrm{Mr} \mathrm{R}$. A. Payne and $\mathrm{Mr}$ J. Beaugié, for allowing us to study their patients, and the nursing staff of the North Middlesex Hospital. In the Department of Medical Physics we thank Mrs J. Buckle and Mrs E. M. Turner for producing the rectilinear scans and Mrs Janet Howe who helped with the ventilation perfusiorf studies.

The ${ }^{81} \mathrm{mKr}$ generators were collected weekly from Dr IWatson's team at the MRC Cyclotron Unit at Hammersmith Hospital. Financial support for purchase of these generators was made by Fisons of Loughborough.

Final thanks are due to Miss Brenda Hedges for preparing the manuscript.

\section{References}

Aukland, P., Coates, G. \& Hirsch, J. (1977) Relationship between postoperative pulmonary embolus and deep venous thrombosis. British Journal of Surgery, 2, 826.

Browse, N.L., Clemenson, G., Bateman, N.T. \& Croft, D.N. (1976) Effect of intravenous dextran 70 and pneumatic leg compression on incidence of postoperative pulmonary embolus. British Medical Journal, 2, 1281.

Browse, N.L., Clemenson, G. \& Croft, D.N. (1974) Fibrinogen-detectable thrombosis in the legs and pulmonary embolism. British Medical Journal, 1, 603.

FAzIO, F. \& JonES, T. (1975) Assessment of regional ventilation by continuous inhalation of radioactive 81 m-krypton. British Medical Journal, 3, 673.

International Multicentre Trial (1977) Prevention of fatal postoperative pulmonary embolism by low doses of heparin-reappraisal of results. Lancet, i, 567.

Kakkar, V.V., Howe, C.T., Nicolaides, A.N., RenNey, J.T.G. \& Clarke, M.B. (1970) Deep vein thrombosis of the leg. Is there a 'high risk' group? American Journal of Surgery, 120, 527.

Kline, A., Hughes, L.E., Campbell, H., Williams, A.. Zlosnick, J. \& LEACH, K.G. (1975) Dextran 70 in prophylaxis of thromboembolism disease after surgery; a clinicall? orientated randomized double blind trial. British Medical Journal, 2, 109. 
Knight, M.T.N. \& Metrevelli, C. (1977) Postoperative pulmonary perfusion defects: their natural history, origin and significance. British Journal of Surgery, 64, 712.

Morrell, M.T. \& Dunhill, M.S. (1968) The postmortem incidence of pulmonary embolism in a hospital population. British Journal of Surgery, 55, 347.

Nicolaides, A.N. \& IRviNG, D. (1975) Clinical factors and the risk of deep venous thrombosis. In: Thromboembolism.
Aetiology, Advances in Prevention and Management (Ed. by Nicolaides, A. N.) Medical and Technical Publishing Co. Ltd, Lancashire, England.

Wessler, S. (1962) Thrombosis in the presence of vascular stasis. American Journal of Medicine, 33, 648.

Williams, I., Lyall, J., Vernon, M. \& CROFT, D.N. (1974) Ventilation-Perfusion lung scanning for pulmonary emboli. British Medical Journal, 1, 600. 